P147 Cost-offset Community-Supported Agriculture plus Nutrition Education Improves Household Food Security and Nutrition Attitudes and Self-efficacy: A Randomized Controlled Trial

Rebecca Seguin-Fowler,PhD, RD, CSCS, r.seguin-fowler@ag. tamu.edu, Texas A\&M AgriLife Research, 600 John Kimbrough Blvd, Suite 512, College Station, TX 77843; Alice Ammerman, DPH, University of North Carolina at Chapel Hill; Karla Hanson, PhD, Cornell University; Stephanie Jilcott Pitts, PhD, East Carolina University; Jane Kolodinsky, PhD, University of Vermont; Grace Marshall, MHS, Cornell University; Emily Morgan, PhD, University of Vermont; Marilyn Sitaker, MPH, MS, The Evergreen State College; Weiwei Wang, BA, University of Vermont

Background: Subsidized or 'cost-offset' CommunitySupported Agriculture (CO-CSA) has the potential to improve food security in low-income households. Furthermore, CO-CSA coupled with tailored nutrition education may affect nutrition knowledge, attitudes, and self-efficacy. To date, many studies of CO-CSA had small samples and no comparison group; few studies incorporated formal nutrition education into CO-CSA.

Objective: To understand the effect of CO-CSA plus education on nutrition knowledge, attitudes, self-efficacy, and food security among low-income families; whether any observed effects are durable beyond the CO-CSA season; and whether intervention dose mediated net effect.

Study Design, Setting, Participants: Farm Fresh Foods for Healthy Kids (F3HK), a randomized controlled trial in New York, North Carolina, Vermont, and Washington (2016-2018) that assigned caregiver-child dyads $(n=305)$ from low-income households into intervention or control. The F3HK intervention included a cost-offset (50\% subsidy) CSA, weekly payments (including SNAPEBT), kitchen equipment, and 9 CSA-tailored education classes.

Measureable Outcome/Analysis: Household food security was measured using the 6-item Short Form of the US Department of Agriculture Food Security Survey Module (FSSM). Caregiver knowledge was assessed by asking recommended cups of fruit and vegetables (FV) per day and FV as a fraction of a dinner plate. The 4-item Negative Cooking Attitude Scale measured caregiver's attitudes towards cooking. The 4-item Self-Efficacy for Eating/ Cooking Fruits and Vegetables Scale measured FV selfefficacy.

Results: Significant net effects were observed for outcomes related to household food security as well as caregiver nutrition attitudes and self-efficacy. Improvements in caregiver attitudes and self-efficacy remained 6 months post-intervention. Weeks of CSA pick-up mediated changes household food security.

Conclusions: CO-CSA plus nutrition education for lowincome caregivers and their children improved household food security and caregiver attitudes and self- efficacy. Attitude and self-efficacy but not food security effects were maintained post-intervention, indicating both the promise and limitations of seasonal food access interventions.

Funding: USDA.

\section{P148 California SNAP-Ed Direct Education and PSE Aggregation}

Brian Petrie, MA, brian.petrie@wellness.phi.org, Center for Wellness and Nutrition, 1750 How Ave, Suite 550, Sacramento, CA, 95825; Alondra Vega-Arroyo, PhD, Center for Wellness and Nutrition; Samantha Trammell, BS, California Department of Social Services; Celeste Doerr, $P h D$, Center for Wellness and Nutrition; Amy DeLisio, $M P H, R D$, Center for Wellness and Nutrition

Background: The Agriculture Improvement Act of 2018 (Farm Bill) has refocused SNAP-Ed reporting to include and prioritize aggregation of direct education and policy, systems, and environmental (PSE) reporting. In federal fiscal year 2016, the USDA Food and Nutrition Service (FNS) Western Region Office instructed CalFresh Healthy Living (CFHL) (California's SNAP-Ed program) to synthesize results from its 4 state implementing agencies (SIAs) and 138 local implementing agencies while reducing the report's overall length. CFHL now reports all SNAP-Ed work from a state perspective by aggregating all data.

Objective: Synthesize and communicate the methods used by CFHL to aggregate and report their SNAP-Ed data that is in accordance with the Farm Bill. This will provide a process that other states can adapt to fit their programs and report their work within the new federal guidelines.

Study Design, Setting, Participants: Direct education: CFHL reviewed all surveys used by SIAs and identified common questions that could be aggregated. Statistical analyses were identified that were appropriate for the data and incorporated effect sizes to measure the size of reported changes. PSE: CFHL used an online reporting system to collect all data, ensuring that all SIAs were collecting the same information which could then be aggregated at the state level.

Measurable Outcome/Analysis: A review of the shifts in methodology CFHL took to aggregate analyses and improve overall research methods to be in line with the Farm Bill and Evidence Act.

Results: Review of outcomes from CFHL's 2018 aggregated analyses, which includes 12 statistically significant direct education findings for MT1 and MT2 indicators.

Conclusions: California has a process for aggregating data that is in line with the Farm Bill that other states can adapt to fit their programs and reporting needs. This may provide a framework for states who may not have evaluation staff with time to think through these processes.

Funding: Supplemental Nutrition Assistance Program Education. 